\title{
Comment percevoir la modélisation en didactique des langues
}

\author{
Jean-Paul Narcy-Combes
}

\section{OpenEdition}

\section{Journals}

Édition électronique

URL : http://journals.openedition.org/asp/1678

DOI : 10.4000/asp. 1678

ISBN : 978-2-8218-0388-6

ISSN : 2108-6354

Éditeur

Groupe d'étude et de recherche en anglais de spécialité

Édition imprimée

Date de publication : 1 décembre 2002

Pagination : 219-230

ISSN : 1246-8185

\section{Référence électronique}

Jean-Paul Narcy-Combes, «Comment percevoir la modélisation en didactique des langues », ASp [En ligne], 35-36 | 2002, mis en ligne le 17 septembre 2010, consulté le 01 mai 2019. URL : http:// journals.openedition.org/asp/1678; DOI : 10.4000/asp.1678

Ce document a été généré automatiquement le 1 mai 2019

Tous droits réservés 


\title{
Comment percevoir la modélisation en didactique des langues
}

\author{
Jean-Paul Narcy-Combes
}

1 La modélisation, thème $\mathrm{du}$ Colloque $2002 \mathrm{du}$ GERAS, est une des questions épistémologiques qui interpellent les chercheurs en didactique des langues. L'objectif de cet article est de montrer qu'une référence plus approfondie à la méthodologie de la recherche en sociologie peut nous donner une dynamique nouvelle, tout autant que le peut un regard moins distant que celui que nous avons en général sur les positions des épistémologues des sciences de la nature.

2 Cet article s'inscrit dans une continuité et poursuit la réflexion entamée dans plusieurs articles déjà publiés dans ASp (Narcy-Combes 2000) et ailleurs (Narcy-Combes1998b entre autres). Les apports nouveaux donnent des outils aux chercheurs de notre domaine pour mieux préciser les positionnements qui conditionnent leurs recherches, la légitimité de celles-ci et leur impact potentiel.

3 L'organisation de cet article reflète plus l'évolution d'une pensée que la construction d'un plan à la logique cartésienne, sans doute parce que la réflexion s'est largement nourrie de la lecture de Damasio, l'auteur de L'erreur de Descartes.

4 Une rapide réflexion bibliographique précèdera quelques définitions qui circonscriront le domaine dans lequel nous nous situons. Il sera alors possible d'aborder la réflexion épistémologique et les problèmes qui l'accompagnent pour le chercheur. C'est donc celuici qui deviendra alors le centre d'intérêt afin de déterminer comment son fonctionnement affecte sa conception de la recherche. Il sera alors possible d'avancer une approche épistémologique qui permet de rester cohérent avec ce qui précède. Cette approche conditionne l'emploi qui peut se faire des divers outils que la méthodologie propose, et impose d'être très explicite sur de nombreux points. Nous en verrons les gains en cohérence et en légitimité, ce qui n'est pas un mince avantage. 


\section{Dette bibliographique}

5 Aller au-delà de notre domaine de recherche en particulier pour mettre en place un module d'épistémologie pour une École doctorale a permis de répondre à des questions que se posait déjà une unité transdisciplinaire de l'Université de Compiègne (COSTECH). La difficulté a toujours été d'intégrer les positions des chercheurs ou des épistémologues d'autres domaines, dont les points de vue étaient topographiquement, pourrait-on dire, très différents des nôtres. Une rencontre particulièrement fructueuse avec la réflexion sociologique a déclenché une réelle réorganisation de nos conceptions en donnant une clé pour lier les réflexions sur le fonctionnement cognitif des individus, les contraintes de notre domaine et les postures avec lesquelles les chercheurs abordent ces contraintes. Le choix de leurs outils, et les biais que ces choix impliquent, trouvaient alors une justification logique, si le chercheur acceptait de prendre le recul nécessaire. La bibliographie reflète donc ce travail. Elle contient de nombreuses références pratiques, en sciences humaines ou en sociologie, car ce sont ces domaines qui, selon notre point de vue actuel, opèrent de la façon la plus éclairante pour la didactique des langues, selon la définition à laquelle nous avons choisi d'adhérer.

\section{Définitions}

Consacrons quelques lignes à des définitions du mot modèle avant de revenir à notre domaine. Du côté des sciences humaines, on peut lire :

Pour décrire, vérifier ou expliquer, prévoir, on peut construire un modèle qui est toujours une simplification de la réalité réduite à des descripteurs : plus précisément, un système de relations entre propriétés [...] construit à des fins de description, d' explication ou de prévision. (Bourdieu et al. $1968: 250)^{1}$

7 Juan (1999) nous rappelle, de plus, que le modèle relève de l'approche explicative de la sociologie, point sur lequel nous reviendrons ultérieurement.

En mathématiques, et/ou dans les sciences à méthodologie mathématique, il en va autrement :

L'espace où la science utilise les modèles s'étend de la représentation presque figurative à celui de la mise en relation de concepts d'un haut degré d'abstraction [...]. Ces modèles permettent, entre autres, l'élaboration de modèles simulables sur ordinateur. Un modèle est alors [...] « éminemment calculable». (Joshua \& Dupin $1993: 15-16)$.

9 Il y a donc une différence notable entre un modèle de l'apprenant en didactique, et un modèle de l'apprenant en informatique. Le second est conçu pour permettre des calculs et opérations afin d'assurer son fonctionnement. Reste à connaître la validité de ce fonctionnement. Différence à ne pas perdre de vue quand on travaille en lien avec des informaticiens qui œuvrent sur les TICE.

10 Cette rapide réflexion sur diverses manières de concevoir ce qu'est un modèle soulève des points sur lesquels nous reviendrons :

- Comment se positionner en ce qui concerne un éventuel principe de réalité ?

- Qu'en est-il des concepts de calcul et de prévisibilité dans notre domaine? 
11 Nous entrons là dans une réflexion épistémologique qui va plus loin que ce que nous avons l'habitude de faire dans nos comptes rendus de recherche et qui néanmoins n'est pas sans conséquence sur la légitimité que ces comptes rendus peuvent avoir.

La conception de la didactique dans laquelle notre groupe se reconnaît s'inscrit dans un vaste courant comme le montre cette définition issue de Manesse et al. (1999) :

La recherche en didactique d'une discipline vise à produire des savoirs susceptibles de faire évoluer l'enseignement/apprentissage de cette discipline en fonction des besoins des élèves et des avancées des connaissances. Elle est tournée vers l'action, et vécue comme une pratique sociale.

Quand nous voyons que la recherche-action peut se définir comme : " a systematic process of investigating practical issues or concerns which arise within a particular social context » (Burns : 20), il nous semble logique d'adhérer au consensus où se retrouvent un ensemble de théoriciens (Manesse, Coste, Dabène, Ellis, et Nunan, pour n'en citer que quelques-uns) qui pensent que la recherche-action est la méthodologie de la recherche la mieux adaptée pour gérer les interrogations que suscite ce domaine.

Il arrive que des recherches en didactique soulèvent des questions dont les réponses ne peuvent être données par la recherche-action. Il s'agit souvent d'incursions dans une des sciences de fondement, sur des points où cette science n'a pas encore apporté de réponses concluantes pour l'action. Inclure de telles recherches en didactique est parfaitement acceptable, si nous nous gardons d'en généraliser les conclusions.

15 La définition de la didactique que nous avons acceptée nous impose de réaliser que nos objets de recherche ne sont jamais des items isolés et/ou isolables, il s'agit d'interaction (s) d'objets dans des systèmes complexes, dont on peut dire que chacun d'entre eux est le résultat d'études dans une, ou plusieurs, des sciences sur lesquelles la didactique se fonde. Il est souvent difficile de décrire de telles interactions en langage naturel, et nous recourrons alors à des tableaux, graphiques ou schémas, auxquels nous donnons souvent le nom de modèle. Avons-nous raison de le faire ? Voilà, en fait, la véritable question à laquelle cet article souhaite se consacrer. Pour ébaucher une réponse à ces questions, il convient de considérer comment dans, le cadre méthodologique qui vient d'être décrit, nous construisons notre objet de recherche.

\section{Construction de l'objet de recherche}

Le travail que le tableau 1 décrit se situe dans la phase initiale d'une recherche-action. Il précède l'action. Dans la mesure où l'action modifiera l'objet, ce cycle se répètera chaque fois qu'il n'y aura plus identité satisfaisante entre l'objet sensible, et l'objet construit d'une part et/ou un décalage trop grand entre la connaissance experte/construite (dans la problématique) et les théories en amont. Ces décalages peuvent provenir soit de la double herméneutique des sciences humaines (les humains lorsqu'ils apprennent comment ils fonctionnent modifient leurs comportements, ce qui modifie les résultats des études initiales), soit du fait que l'action a pour objectif de modifier l'objet. Rappelonsnous que des références à la complexité nous manquent encore ici. 
Tableau 1. Construction de l'objet recherche

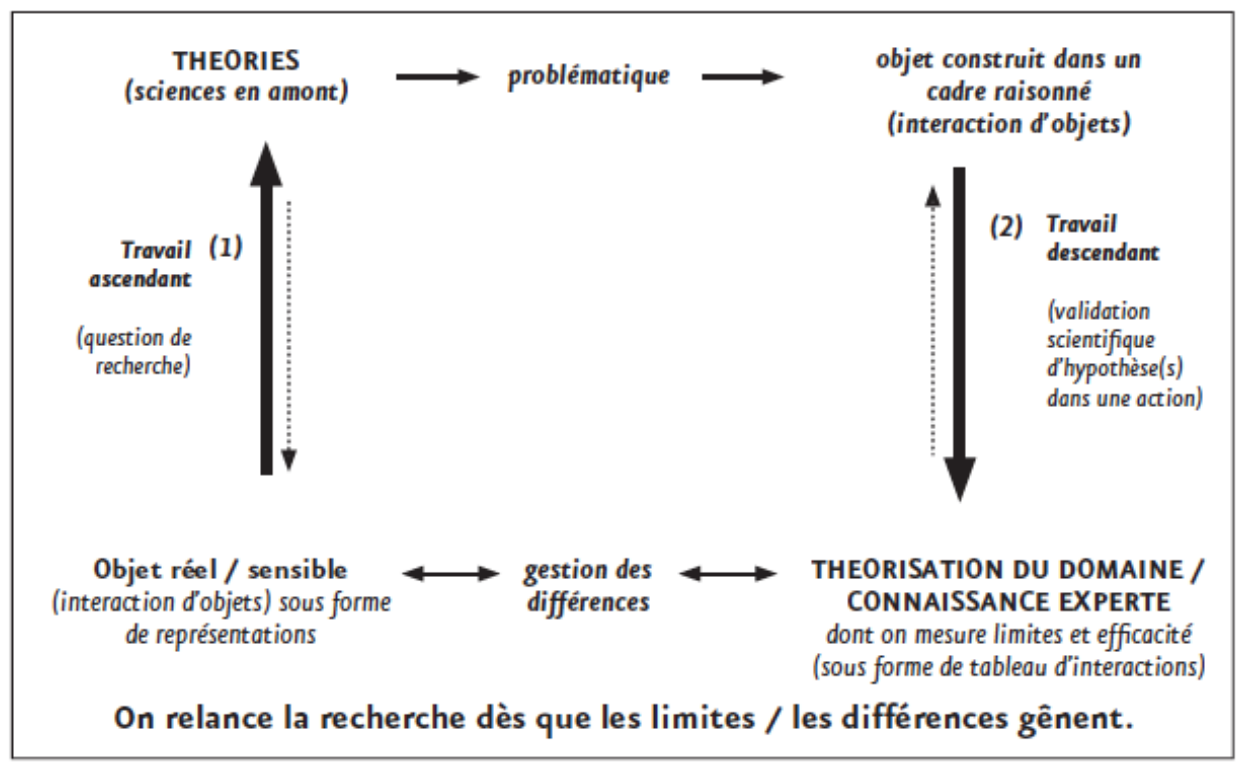
en découlent.

Cette représentation, qui remplace et complète celle publiée dans ASp 27-30, s'insère dans la phase initiale de la colonne recherche du cycle de la recherche-action (Narcy-Combes 1998b). Les changements les plus notoires concernent la compréhension du fait que notre objet de recherche est en fait une interaction d'objets, interaction complexe dans laquelle le contrôle des variables est rarement en notre pouvoir.

Initialement, pour chacun de nous, l'apparence de l'objet sensible (qui est ici une interaction d'objets) est le résultat de notre regard conditionné et des constructions auxquelles nos représentations initiales nous conduisent. Le travail ascendant va consister à le déconstruire en éléments stables et pertinents que l'on pourra confronter aux multiples théories des diverses sciences en amont. Ce travail ne saurait se faire de façon totalement naïve, puisque nous avons eu accès à une ou plusieurs de ces théories, il est donc plus ou moins descendant, en parallèle. Le chercheur devra mesurer les biais qui

De la confrontation aux diverses théories va naître l'objet construit. Cette construction implique que, parmi des théories parfois divergentes, nous nous positionnions (notre problématique est justement l'ensemble théorique dans lequel nous choisissons d'œuvrer). Notre problématique nous amène à construire un objet dont il importe de nous rappeler qu'il aurait pu être construit différemment par un autre chercheur.

Le travail descendant consiste à vérifier la validité de notre construction de l'objet et de son fonctionnement, ce qui nous amène à mettre en place un dispositif de recueil de données dans des systèmes d'apprentissage/ensemble d'actions où nous n'avons qu'un très faible contrôle des variables qui nous permettront de mesurer la validité de nos hypothèses. Ce travail ne saurait avoir lieu sans qu'un travail ascendant ne s'effectue en parallèle. Travail qui ne peut qu'influer plus ou moins implicitement sur nos hypothèses et leur opérationnalisation.

21 Les résultats de notre travail de validation serviront à consolider la théorisation de notre domaine et à augmenter la connaissance experte, s'il y a diffusion vulgarisatrice. Souvent, la complexité de nos conclusions nous conduit à les proposer sous formes de tableaux. Ces 
tableaux ne représentent que la façon dont nous avons compris le fonctionnement des interactions d'objets que nous avons étudiées, dans un contexte donné. Il pourrait paraître hâtif de les appeler modèles. Pourtant, dans la mesure où nous avons à agir, nous le ferons en nous référant à ces tableaux, et ils fonctionneront alors comme pourraient le faire des modèles. Du rôle d'explicitation de ce que nous avons compris, nous passerons à un rôle explicatif et prédictif. Le tout est de le savoir et d'en mesurer les conséquences. Notre travail ne s'arrête pas là. Il nous restera à vérifier si l'objet construit est identique à l'objet sensible et si la théorisation du domaine est en accord avec les conclusions des sciences en amont. Si des incohérences apparaissent, il nous faudra relancer un processus de recherche.

Un retour en arrière s'impose maintenant sur la façon dont nous élaborons notre problématique.

\section{Des théories à une problématique}

Revenons maintenant à la construction d'une problématique (Narcy-Combes 2000a), qui, dans notre domaine, est particulièrement complexe.

Tableau 2. Comment sélectionnons-nous les sciences et les théories : notre monothéisme ou notre polythéisme sont-ils rationnels?

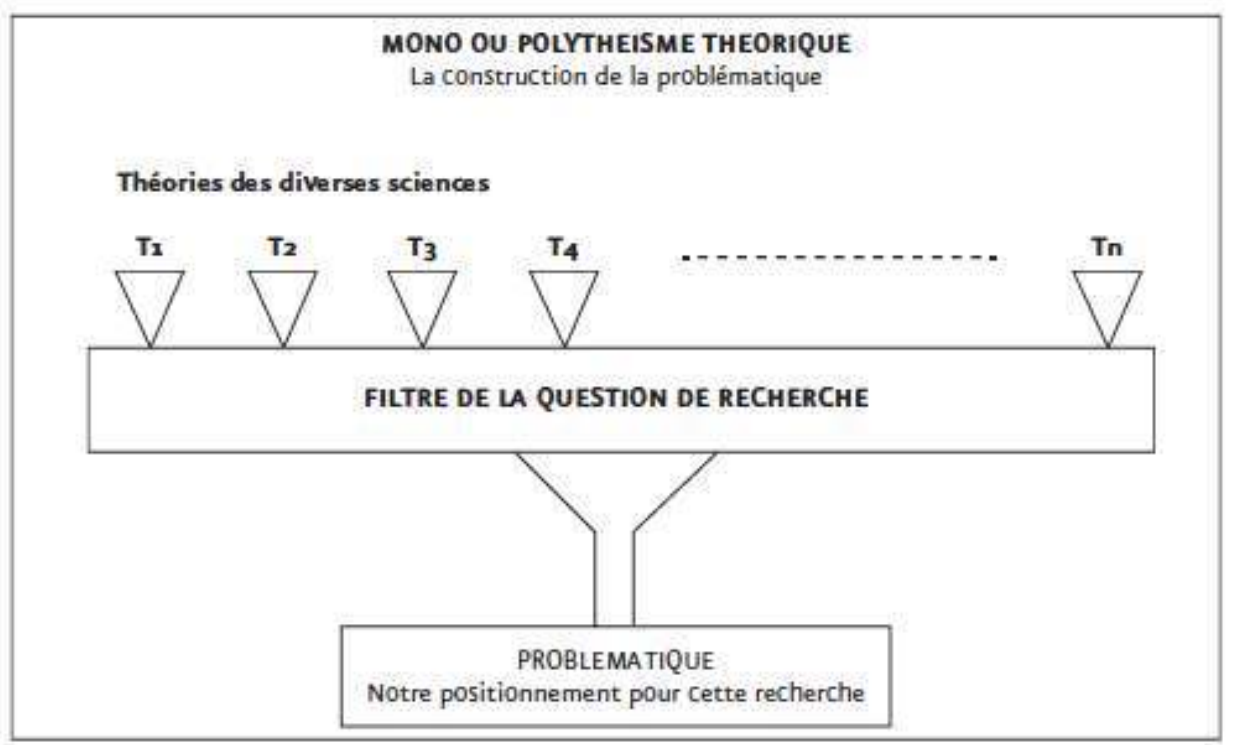

Ce qui nous arrête maintenant n'est plus la technique des entonnoirs (étudier les diverses théories en fonction des besoins et limites de notre contexte de recherche, cf. NarcyCombes 2000a), mais ce qui justifie nos choix et nos positionnements. Pour le comprendre, nous avons été progressivement conduits à travailler sur les conceptions qui sont actuellement avancées en ce qui concerne la construction de la connaissance humaine. Il nous parait possible d'affirmer maintenant que la recherche nous permet de passer d'une rationalité conditionnée à une rationalité limitée par la prise de conscience de notre besoin constant de recul. 


\section{Positionnement cognitif}

Toute donnée (ou ensemble de données), qui peut activer nos capacités sensorielles, éveille, quand nous entrons en contact avec elle, une sensation qui ne relève pas de notre conscient. Cette sensation est « conditionnée » par notre passé, mais également par l'état émotif du moment et l'effet du contexte social sur nous. Notre perception des données dépend donc de cela. Nous ne verrons, lirons, entendrons, vivrons, que ce que nous sommes conditionnés à voir, lire, entendre, vivre, etc., à ce moment-là (flèche 1 tableau 3). Les émotions relèvent de notre physiologie et non de notre affectif, ni de notre cognitif. Elles génèrent des réactions affectives ou conatives (motivation), en fonction de notre vécu, et ces réactions nous conduiront à une réaction cognitive qui pourrait être différente chez un autre sujet.

Tableau 3. Positionnement cognitif

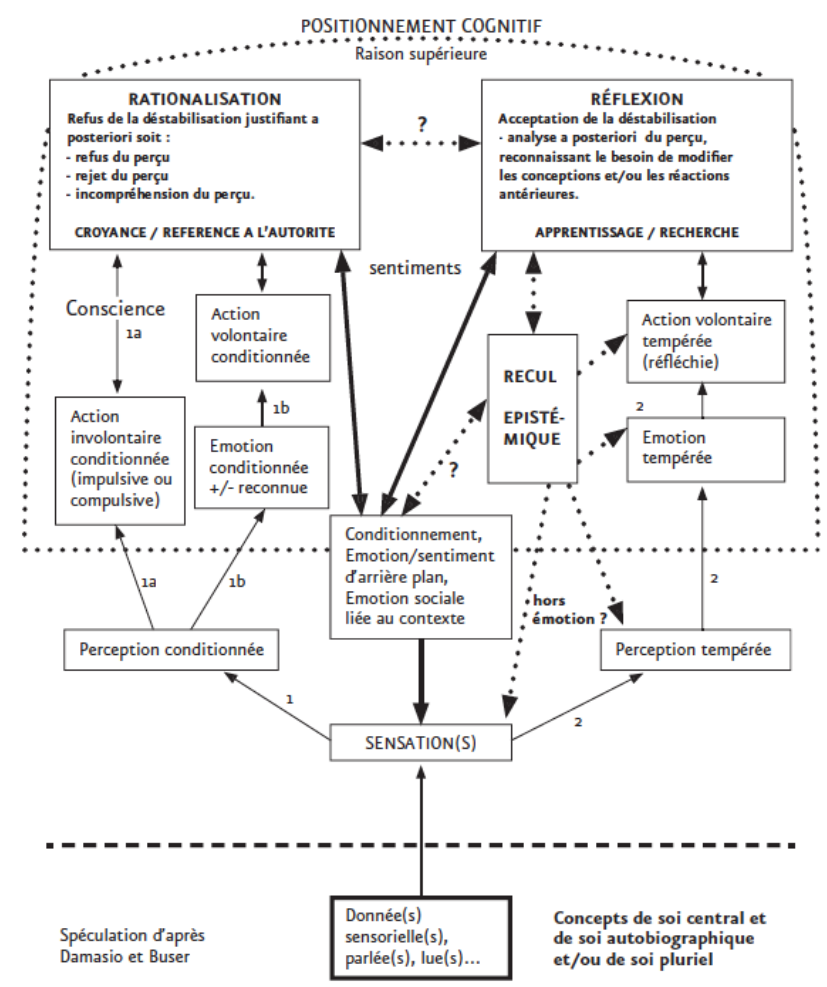

Cette perception, ce vécu des données, agit, disons, en générant une émotion (un jeu d'émotions) qui peut avoir deux effets. Soit, cette émotion enclenche un ensemble complexe d'actions quasi automatiques (a course of action) (flèche 1a), soit elle nous conduit à une prise de décision, conditionnée mais volontaire, c'est-à-dire consciente mais sous cette forme : «je sais que je ressens ceci, je n'ai qu'un choix d'action que je ne discute pas » (flèche $1 b)$.

Si la perception reste semi-consciente, l'action, devient consciente, même si elle est automatique. S'il nous est demandé de raisonner après un tel cheminement, nous rationaliserons (justifierons a posteriori), soit en refusant le perçu réel pour maintenir notre perçu conditionné, soit en rejetant le perçu réel, soit en manifestant notre incompréhension («je ne comprends rien », «je ne vois pas ce que vous voulez dire», 
etc.), soit d'autres manières qui toujours auront une part de logique (résultat des parcours fléchés 1a ou 1b). Dans un cas comme dans l'autre, on comprend qu'il ne saurait $\mathrm{y}$ avoir apprentissage. Pour qu'il y ait apprentissage, il est nécessaire qu'une déstabilisation du conditionnement ait lieu (on retrouve là des notions de la didactique d'aujourd'hui).

Pour faciliter l'apprentissage, Buser postule la mise en place d'un système métacognitif d'autocontrôle. Des chercheurs en sociologie, comme Bourdieu ou Morin, ont eu cette intuition de leur côté. Tout comme les didacticiens qui avancent la nécessaire mise en place d'une réflexion métacognitive. Dans la mesure où les phénomènes relationnels qui accompagnent l'apprentissage d'une langue (en ce qui concerne la communication et l'interculturel) relèvent de cette réflexion, si nous tentons d'intégrer les diverses théories psychologiques ou psychanalytiques dans un modèle temporaire, il parait possible de postuler qu'il serait pertinent que chacun mette en place un recul conscient. Ce recul favoriserait la mesure plus ou moins juste de l'écart qui existe entre ce que notre conditionnement nous fait vivre, et ce qui s'est passé pour d'autres. Il fonctionnerait à chaque niveau, émotions, action et rationalisation ou réflexion (parcours fléché 2). Je suis tenté d'ajouter la qualification d'épistémique à un tel recul pour montrer à quel point il est constitutif de notre personnalité apprenante.

Plus ou moins consciemment, toute personne qui apprend se sert de ce recul à la façon de monsieur Jourdain. Son rôle est explicité dans la partie gauche. Pour le moment, nous gérons apprentissages et vie sans prendre la mesure de l'importance du rôle et du fonctionnement de ce recul. Bien souvent nous voulons, en plus, être complètement dégagés de tout irrationnel en nous. Ce qui signifierait que notre recul est complètement efficace. Cette efficacité absolue est difficile à concevoir même si nous rendons plus conscient le fonctionnement de ce recul. Ce recul nous permet(trait) de tempérer les émotions, modifiant ainsi partiellement les effets d'une perception conditionnée. Il favorise(rait) donc, ensuite, la prise de décisions plus mesurées. Si nous acceptons la déstabilisation nous pouvons modifier ou affiner le perçu de départ. Ce travail cognitif facilitera notre reconnaissance de la nécessité de modifier nos conceptions et réactions antérieures. Le recul nous permet donc d'accepter d'apprendre. Il convient de se rappeler qu'une telle conception repose sur des données neurophysiologiques observables. Il est de ce fait plus difficile qu'auparavant de récuser de telles données et de maintenir des comportements personnels, ou des pratiques enseignantes et apprenantes d'autrefois.

31 Ce qui relève de l'inconscient (cf. Buser) dans ces théories ne relève pas d'un inconscient tel que la psychanalyse le conçoit, mais si nous sommes dominés par un conditionnement (conditionnement qui ici n'est pas une référence à Skinner, mais à Laborit, par exemple), pourquoi ne pas le prendre en compte? Suivant que nous fonctionnons par rationalisation ou par réflexion, nous suivons un cheminement épistémologique radicalement différent comme nous allons maintenant le voir.

Ceci explique comment nous pouvons passer d'un conditionnement rationalisé à une rationalité limitée. Il reste à expliquer comment nous pouvons maintenant appréhender ce qui contribue à donner au chercheur sa posture. 


\section{La posture du chercheur}

Pour tout chercheur qui adhère aux théories synthétisées dans le point 5 , son conditionnement personnel et culturel joue un rôle non négligeable dans la construction de sa posture.

Il nous semble possible, en fonction d'autres études (Barbot \& Camatarri entre autres), d'ajouter ses valeurs, ses goûts, ses visées, ses ressources et ses contraintes.

Le recul permettra à chacun de mesurer ce que représente chacun de ces points pour lui, comment ces derniers influent sur sa recherche, et quel degré de cohérence qu'il atteint. Dans une culture où la recherche quantitative est valorisée, par exemple, un chercheur qui souhaite faire carrière (visée) peut être conduit à choisir une approche de ce type, tout en se connaissant une approche très humaniste (au sens Rogérien) et très ascendante des relations humaines. Il y aura alors un manque de cohérence au niveau méthodologique, et donc au niveau de la légitimité des résultats. C'est la rencontre avec la sociologie qui nous a sensibilisés à ce problème.

\section{Les postures en sociologie}

Les sociologues semblent d'accord pour relever trois grandes postures de base, tout en n'en retenant que deux principales, si leurs propos ont été bien compris.

\subsection{La posture explicative (tradition de Durkheim)}

Elle est objectiviste et le chercheur s'intéresse aux observables et non à ce qui est intérieur aux individus. Elle est descendante, à partir d'une théorie, elle formule des hypothèses et cherche à les valider.

7.2. La posture compréhensive (tradition de Weber)

38 Elle est subjectiviste et cherche à comprendre ce qui meut les individus. Elle recherche le sens que les personnes donnent à leur action. Elle se veut ascendante (elle part de données pour comprendre les phénomènes sociaux).

7.3. Une posture plus interactioniste (tradition école de Chicago)

Dans cette posture, les chercheurs ne théorisent pas généralement, mais opèrent en fonction des contextes et des problèmes. Le rapport avec le point 7 est évident. Un conditionnement personnel ou culturel peut expliquer que l'on se reconnaisse plus dans une posture ou une autre. Comprendre pourquoi et comment (recul) rendra plus légitime la position prise et permettra une plus grande cohérence. Le lien entre posture et méthodologie de recherche est évident en sociologie. Avant de nous y arrêter, il nous reste à nous positionner sur le sens que peuvent avoir les concepts de vérité et de réalité. Ce positionnement nous permettra de mieux concevoir comment organiser les paramètres qui gouvernent les options épistémologiques d'une recherche, et de proposer un cadre qui permet de nous assurer de la cohérence méthodologique de cette recherche. 


\section{Positionnement épistémologique}

Pour les épistémologues,

- Le réalisme pour qui la théorie décrit ce à quoi le monde ressemble. Ce concept inclut la notion de vérité.

- L'instrumentalisme qui affirme que les théories sont conçues comme des instruments pour relier des séries d'états observables.

41 Selon Chalmers, la vision réaliste serait plus motivante pour les chercheurs (il s'agit d'une opinion difficilement justifiable par observation, mais elle est séduisante). Comme le réalisme lui paraît difficilement tenable au niveau épistémologique, il propose le concept de réalisme non figuratif. Selon lui, le but de la physique (d'une science) est d'établir des limites à l'application des théories actuelles et de développer des théories qui soient applicables au monde avec le plus grand degré d'approximation dans une grande variété de circonstances. Ceci rejoint d'autres points de vue selon lesquels il n'est pas possible d'établir une concordance iconique entre la représentation et la réalité (cf. Barbot \& Camatarri 1999 : 53).

Le réalisme non figuratif est réaliste car, d'une part, il contient l'hypothèse que le monde sensible (l'apprenant dans une interaction pour nous) est ce qu'il est indépendamment de notre connaissance. D'autre part, il contient l'hypothèse que, dans la mesure où les théories sont applicables au monde sensible (à l'apprenant dans une interaction), elles le sont toujours, en situation expérimentale ou non.

Il est non figuratif car il ne s'agit pas d'une théorie de la correspondance de la vérité avec les faits. En effet, nous n'avons jamais accès au monde indépendamment de nos théories (ce qui est contre-intuitif).

44 Cette théorie a l'avantage de résoudre tout conflit diachronie/synchronie. Dans la diachronie, il y a quête de la vérité. Dans la synchronie, il peut y avoir des aléas de parcours. Ce fait justifie le polythéisme dans la synchronie, dès lors qu'il n'est pas possible d'atteindre un point de vue unifié. Si l'idéologie consiste à développer des théories pour convaincre les autres que l'on a raison (cf. Cyrulnik), cela ne semble pas le cas ici, tout en soulevant un authentique débat.

Nous sommes maintenant en mesure de comprendre comment les approches de recherche se mettent en place et les conséquences que cela implique au niveau de la méthodologie.

\section{Nos approches et leurs conséquences méthodologiques}

Commençons par le tableau 4 qui s'appuie sur un modèle de Juan, complété par les concepts issus de Chalmers. 
Tableau 4. Approches et horizons de la méthode en sciences humaines d'après S. Juan et Chalmers)

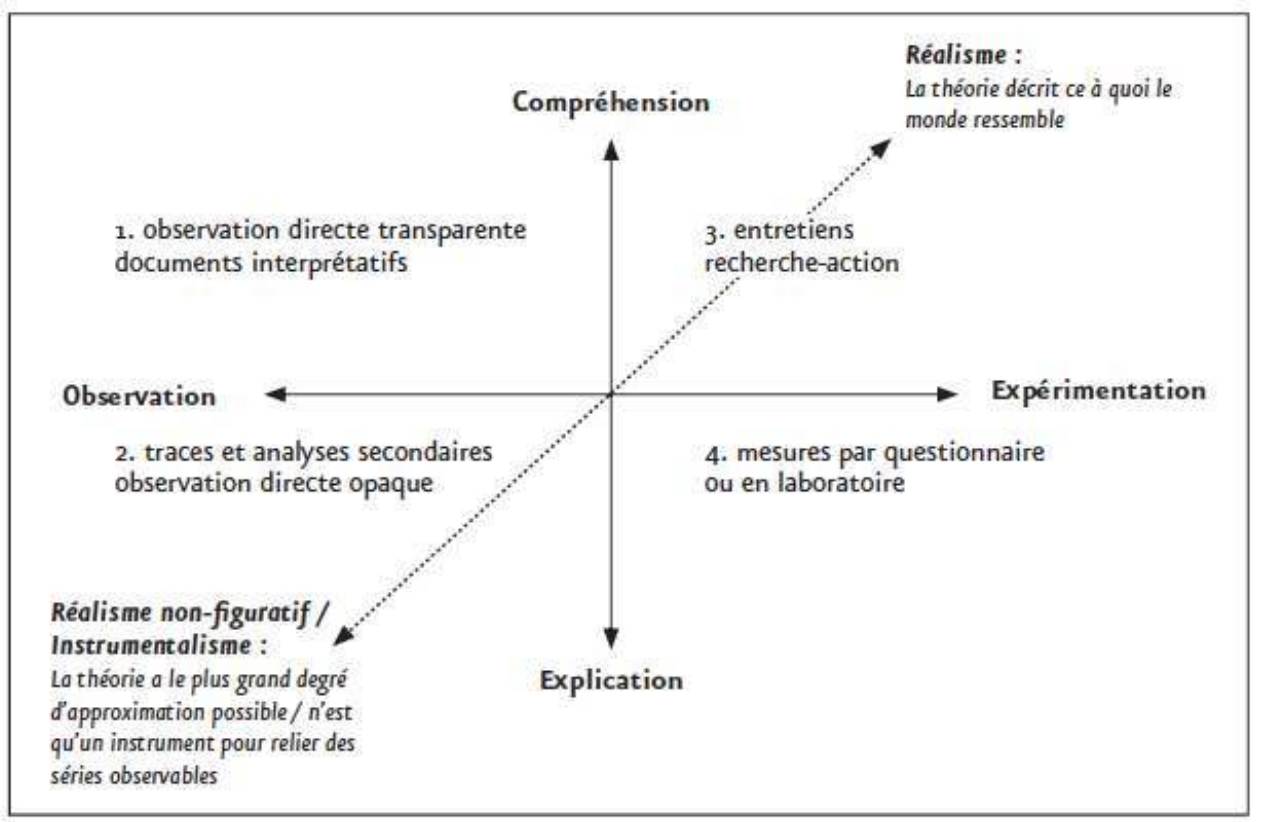

Le positionnement que nous avons sur le réalisme, le réalisme non figuratif ou l'instrumentalisme, conditionnera l'autorité que nous attribuons aux résultats de la recherche et à nos représentations sur leur stabilité. Notre posture complétera ce qui précède, c'est elle qui guidera nos choix méthodologiques, comme le montre le tableau.

De nombreux chercheurs pensent que les postures et les approches se complètent, et cela paraît rassurant, encore faut-il que cela se fasse en toute cohérence et en toute clarté. En ce qui concerne la didactique, il nous reste à résoudre un dilemme. Si des chercheurs, avec lesquels notre groupe est en accord, pensent que la recherche-action en est la méthodologie constitutive, cela vient-il de leur posture ? Il convient alors de le dire. Ou cela est-il une vérité ? Dans ce cas, nous serions en contradiction avec ce qui précède...

\section{Conclusion}

De ce qui précède nous pouvons conclure que :

1. Connaître sa posture permet d'être cohérent dans sa démarche, (moins de rationalisation ou d'intellectualisation).

2. Il nous est possible de mieux gérer la cohérence de la démarche et des outils.

3. Il existe un jeu de complémentarité (de la compréhension à l'explication, ou à l'interaction, etc.) qu'il nous sera utile de mieux comprendre.

4. Nos conclusions de recherche auront ainsi une plus grande légitimité.

Cet article nous a permis de voir que la nature de notre domaine de recherche fait de notre objet de recherche une interaction d'objets dans des systèmes complexes, mais la complexité au sens de Morin et de Le Moigne n'est pas abordée. Il semblerait que cet objet nous impose partiellement notre posture dans la mesure où la recherche-action s'affirme comme mode de recherche constitutif (ce qui reste à approfondir). Si nous adoptons une approche compréhensive, qui est celle qui est cohérente en recherche-action, comme 
nous avons besoin de tableaux pour présenter notre compréhension des interactions sans trop dépendre du langage humain dont la précision serait source de confusions, nous avons envie de les appeler modèles. Cela nous place dans la tradition explicative, qui n'est pas celle qui correspond à la recherche-action. Ces tableaux nous aident à comprendre, mais il est difficile de s'en servir pour prédire ou calculer. S'ils fonctionnent comme modèles lors de la mise en place d'action, il convient d'être vigilant afin de les modifier dès que l'action révèle un point faible. Dans la mesure où notre domaine reste celui de la praxéologie, nous pouvons penser que la complémentarité des approches et des postures se fait dans la complémentarité avec les sciences de fondements. Cette position ressemble trop à une pirouette facile pour s'en contenter, mais pour le moment il nous faut bien l'accepter.

\section{BIBLIOGRAPHIE}

Barbot, M. J. et G. Camatarri. 1999. Autonomie et apprentissage. L'innovation dans la formation. Paris : Presses Universitaires de France.

Blanchet, A. et A. Gotman. 2001. L'enquête et ses méthodes : l'entretien. Paris : Nathan.

Block, D. 1999. « Who framed SLA research?' ». In Cameron L. \& G. Low, Researching and Applying Metaphor. Cambridge : Cambridge University Press, 135-148.

Bourdieu P. et al. 1968. Cité dans S. Juan. 1999. Méthodes de recherche en sciences sociohumaines. Paris : Presses Universitaires de France.

Bourguignon, C. 2001. « La communication langagière : évaluation, médiation, re-médiation ». Communication au Colloque du GERAS, Bordeaux 2, 29-31 Mars 2001.

Buser, P. 1999. Cerveau de soi, cerveau de l'autre. Paris : Odile Jacob.

Burns, A. 1995. Presses Universitaires de France. Cambridge : Cambridge University Press.

Cabin, P. et J.-F. Dortier (dir.). 1999. La sociologie. Auxerre : Sciences Humaines.

Chalmers, A. F. 1987. Qu'est-ce que la science? Paris : La Découverte.

Cyrulnik, B. 1983. Mémoire de singe et parole d'homme. Paris : Hachette.

Damasio, A. R. 1995. L'erreur de Descartes, la raison des émotions. Paris : Odile Jacob.

Damasio, A. R. 1999. Le sentiment même de soi. Paris : Odile Jacob.

Dortier, J.-F. 1999. « La société dans la tête ». In Le cerveau et la pensée, Auxerre : Sciences Humaines.

Elias, N. 1993. Engagement et distanciation. Paris : Fayard.

Ellis, R. 1997. SLA Research and Language Teaching. Oxford : Oxford University Press.

Joshua, S et J.-J. Dupin. 1993. Introduction à la didactique des sciences et des mathématiques. Paris :

Presses Universitaires de France. 
Juan, S. 1999. Méthodes de recherche en sciences socio-humaines. Paris : Presses Universitaires de France.

Kaufmann, J-C. 2001. L'entretien compréhensif. Paris : Nathan.

Kaufmann, J-C. 2001. Ego, pour une sociologie de l'individu. Paris : Nathan.

Kuhn, T. 1970. The Structure of Scientific Revolutions. Chicago, IL : University of Chicago Press.

Laborit, H. 1994. La légende des comportements. Paris : Flammarion.

Lakatos, I. 1984. Preuves et réfutations, Essai sur la logique de la découverte mathématique. Paris : Hermann.

Lamizet, B. 1992. Les lieux de la communication. Liège : Éditions Mardaga.

Larsen-Freeman, D. et M. H. Long. 1991. An Introduction to Second Language Research. Londres : Longman.

Le Moigne, J-L. 1980. La modélisation des systèmes complexes. Paris : Bordas.

Linard, M. 1996. Des machines et des hommes. Paris : L'Harmattan.

Manesse, D., D. Coste et M. Dabène. 1999. « Points de vue sur la recherche action ». Repères 20.

Morin, E. 1977. La Méthode 1 : La nature de la nature. Paris : Seuil.

Narcy, J.-P. 1997. « Vers une pratique théorisée et humaniste ». In Ginet et al., Du laboratoire de langues à la salle de cours multi-médias. Paris : Nathan, 45-90.

Narcy, J.-P. 1998a. " La problématique Action Research/Recherche action et le travail coopératif ». ASp 19-22, 229-238.

Narcy, J.-P. 1998b. « Le Monde à notre portée ». Études de Linguistiques Appliquées 112, 435-448.

Narcy, J.-P. 1998c. « Représentations, apprentissages et supports multimédias ». Cahiers de l'APLIUT 17/3, 14-24.

Narcy-Combes, J.-P. 2000. « Épistémologie et méthodologie de la recherche dans le secteur LANSAD : qu'apporterait une harmonisation des pratiques ?». ASp 27-30, 243-251.

Narcy-Combes, J.-P. 2001. « La recherche-action en didactique des langues : apprentissage, compagnonnage ou évolution libre? ». Cahiers de l'APLIUT 21/2, 39-52.

Narcy-Combes, J.-P. (dir). 2002. Actes du Colloque Recherche Action, La Rochelle, Mars 2001, Revue de l'IUFM de Poitou-Charentes.

Nunan, D. 1992. Research Methods in Language teaching. Cambridge : Cambridge University Press.

Popper, K. 1999. All Life is Problem Solving. Londres : Routledge.

Quivy, R. et L. VanCampenhoudt. 1988. Manuel de recherche en sciences sociales. Paris : Dunod.

Resweber, J-P. 1995. La recherche action. Paris : Presses Universitaires de France.

Seliger, H. W. et L. Shohamy. 1989. Second Language Research Methods. Oxford : Oxford University Press.

Singly, F. de. 2001. L'enquête et ses méthodes : le questionnaire. Paris : Nathan. 


\section{NOTES}

1. Mes italiques

\section{RÉSUMÉS}

En fonction des domaines de recherche dans lequel il est employé, le concept de modèle prend des définitions assez divergentes. Si on admet que la recherche-action est la forme de recherche la plus adaptée à la didactique des langues et que l'objet de recherche est en fait une interaction d'objets, on est confronté à un dilemme: puisque la recherche-action relève d'une posture compréhensive et que la modélisation relève d'une posture explicative, il semble peu logique de modéliser en didactique des langues de façon généralisante. Pourtant, la complexité même de l'objet de recherche impose au chercheur de décrire comment il comprend son fonctionnement sous forme de tableaux d'interactions, auxquels il serait préférable de ne pas donner le statut du modèle mathématique ou informatique. L'article tentera de déterminer alors ce qui conditionne le chercheur, et comment cela influence sa posture de chercheur. Il conclura en montrant comment le chercheur peut maintenant expliciter la cohérence de sa démarche et en mesurer les limites et les biais.

The concept of model is defined in diverging ways according to the various domains in which it is referred to. If action research is seen as the best adapted form of research when language didactics is concerned, and if the object of research is envisaged as an interaction of objects in this domain, then the researcher faces a dilemma: action research results from a comprehensive approach whereas modelisation results from an explanatory approach. As a consequence it may seem illogical to design models in language didactics. And yet, the very complexity of the object of research leads researchers to describe the way they understand the interactions under investigation in a visual, schematic way. It may however be preferable to refrain from referring to the result as if it were a mathematical or computer model. The paper will then try to determine what researchers are conditioned by and how this influences each individual approach to research. The conclusive paragraphs will be devoted to how researchers can now explicit how coherent individual approaches are and what their limitations and biases are.

\section{INDEX}

Keywords : action research, comprehensive approach, epistemic distanciation, epistemology, explanatory approach, methodology, model, posture

Mots-clés : approche compréhensive, approche explicative, épistémologie, méthodologie, modèle, posture, recherche-action, recul épistémique 


\section{AUTEUR}

\section{JEAN-PAUL NARCY-COMBES}

Jean-Paul Narcy-Combes est Professeur à l'Université de La Rochelle et appartient au CERCI de Nantes. Ses recherches portent sur les stratégies d'apprentissage et la théorisation de la pratique, l'épistémologie et la méthodologie de la recherche, en lien avec les TIC. Il a coordonné les travaux qui ont conduit au Livre blanc sur l'évaluation en Langues (UPLEGESS), et a été consultant scientifique du projet DCL. jean-paul.narcy-combes@wanadoo.fr 